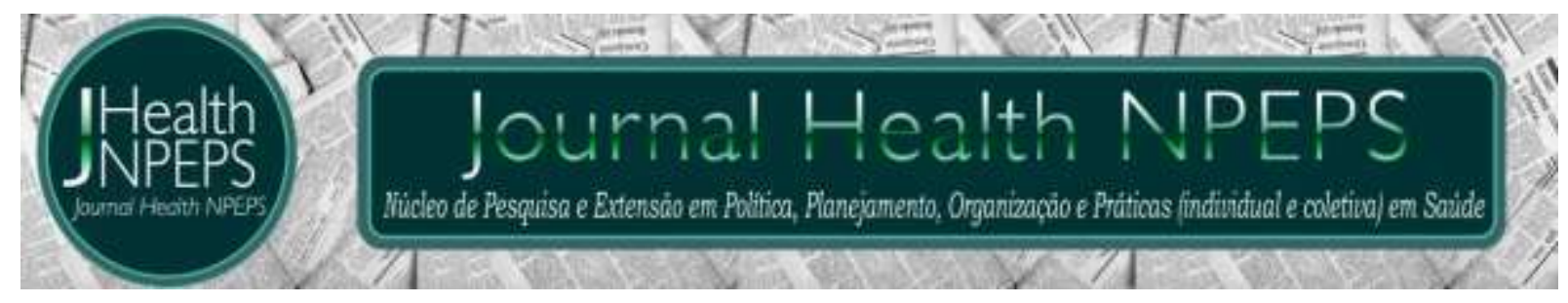

http://dx.doi.org/10.30681/252610103697

ARTIGO ORIGINAL

\title{
Incertidumbre y calidad de vida en pacientes con hipertensión
}

\author{
Uncertainty and quality of life in patients with hypetension \\ Incerteza e qualidade de vida em pacientes com hipertensão
}

\begin{abstract}
Alejandra Hernández Nicio ${ }^{1}$, Julio Cesar Chávez Vásquez², Juana Mercedes Gutiérrez Valverde ${ }^{3}$, José Alfredo Pimentel Jaimes ${ }^{4}$, Juan Yovani Telumbre Terrero $^{5}$, Liliana Leticia Juárez Medina ${ }^{6}$, Geu Mendoza Catalán ${ }^{7}$
\end{abstract}

\begin{abstract}
RESUMEN
Objetivo: determinar la correlación entre el nivel de incertidumbre, la calidad de vida y la presión arterial en adultos con hipertensión de Santiago Pinotepa Nacional Oaxaca. Método: el diseño del estudio fue transversal y correlacional. La población de interés fueron pacientes hipertensos de 20 años de edad y más, con diagnóstico mínimo de 6 meses y que no presenten otras enfermedades crónicas. El muestreo fue no aleatorio por conveniencia. La recolección de datos se realizó por medio de visitas domiciliarias. Resultados: la muestra estuvo integrada por 119 pacientes, la edad promedio fue de 65.7 años $(D E=14.7)$. En la muestra
\end{abstract}

\footnotetext{
${ }^{1}$ Enfermera. Pasante de Licenciatura del Servicio Social. Hospital General de Puerto Escondido. Puerto Escondido, Oaxaca, México. E-mail: ale-1994-@ hotmail.com ORCID ID: http://orcid.org/0000-0001-6768-0635 ${ }^{2}$ Enfermero. Pasante de Licenciatura del Servicio Social. Centro de Salud Cacahuatepec. Puerto Escondido, Oaxaca, México. E-mail: juliitoo12@gmail.com ORCID ID: http://orcid.org/0000-0002-7744-887X

${ }^{3}$ Enfermera. Doctora en Ciencias de Enfermería. Profesor de tiempo completo. Facultad de Enfermería, Universidad Autónoma de Nuevo León. Monterrey, Nuevo León, México. E-mail: juana.gutierrezv@uanl.mx ORCID ID: https://orcid.org/0000-0001-9506-5947

${ }^{4}$ Enfermero. Maestro en Ciencias de Enfermería. Profesor Investigador. Facultad de Enfermería, Universidad Autónoma de Baja California. Mexicali, Baja California, México. E-mail: alfredo.pimentel@uabc.edu.mx ORCID ID: https://orcid.org/0000-0003-4384-8370

${ }^{5}$ Enfermero. Maestro en Ciencias de Enfermería. Profesor Investigador de tiempo completo. Facultad de Ciencias de la Salud. Universidad Autónoma del Carmen. Ciudad del Carmen, Campeche, México. E-mail: jytt @ hotmail.com ORCID ID: http://orcid.org/0000-0003-4384-8370

${ }^{6}$ Enfermera. Doctora en Ciencias de Enfermería. Profesora de tiempo completo. Facultad de enfermería, Universidad Autónoma de Tamaulipas, Nuevo Laredo, México. E-mail: lilianajuarezm@gmail.com ORCID ID: https://orcid.org/0000-0002-9656-4198

${ }^{7}$ Enfermero. Doctor en Ciencias de Enfermería. Responsable de innovación y desarrollo docente. Universidad Autónoma de Baja California. Baja California, México. E-mail: geu.mendoza@uabc.edu.mx ORCID ID: http://orcid.org/0000-0002-5061-2457 Autor principal - Endereço para correspondência: Calle G S/N, Nueva, 21100 Mexicali, B.C., México. Tel: 6865518200
}

Este artigo está licenciado sob forma de uma licença Creative Commons Atribuição 4.0 Internacional, que permite uso irrestrito, distribuição e reprodução em qualquer meio, desde que a publicação original seja corretamente citada. 
Hernández-Nicio A, Chávez-Vásquez JC, Gutiérrez-Valverde JM, Pimentel-Jaimes JA, Telumbre-Terrero JY, Juárez-Medina LL, et al.

predominó el sexo femenino, casado y analfabeta, el $73.9 \%$ reportó una incertidumbre baja, el $50.4 \%$ señaló tener una muy buena calidad de vida y el $52.9 \%$ presentó cifras de presión sistólica $>140 \mathrm{mmHg}$. La calidad de vida se correlacionó significativamente con la presión arterial. Conclusión: es necesario realizar detección de la incertidumbre en los pacientes para dirigir intervenciones individualizadas con la finalidad de generar un afrontamiento positivo, favorecer la calidad de vida y coadyuvar a mejorar el control de la presión arterial en esta población.

Descriptores: Incertidumbre; Calidad de vida; Hipertensión.

\section{ABSTRACT}

Objective: to determine the correlation between the uncertainty level, quality of life and blood pressure in adults with hypertension of Santiago Pinotepa Nacional, Oaxaca. Method: it was a cross-sectional and correlational study. The population of interest were hypertensive patients aged 20 years and over, with a minimum diagnosis of 6 months and no other chronic diseases. Sampling was not random sampling by convenience. Data gathering was made by home visits. Results: the sample consisted of 119 patients, the average age was 65.7 years old $(S D=14.7)$. Female, married and illiterate predominated in the sample, 73.9\% reported low uncertainty, 50.4\% reported very good quality of life and $52.9 \%$ had Systolic BP > $140 \mathrm{mmHg}$. Quality of life is significantly correlated with blood pressure. Conclusion: it is necessary to make an identification of uncertainty on patients to manage personal interventions with the purpose of creating a positive confrontation, favor the quality of life and contribute improving the management of blood pressure among this population.

Descriptors: Uncertainty; Quality of Life; Hypertension.

\section{RESUMO}

Objetivo: determinar a correlação entre o nível de incerteza, qualidade de vida e pressão arterial em adultos com hipertensão de Santiago Pinotepa Nacional Oaxaca. Método: o desenho do estudo foi transversal e correlacional. A população de interesse eram hipertensos com 20 anos ou mais, com diagnóstico mínimo de 6 meses e sem outras doenças crônicas. A amostragem foi não aleatória por conveniência. A coleta de dados foi realizada por meio de visitas domiciliares. Resultados: A amostra foi composta por 119 pacientes, a idade média foi de 65,7 anos (DP $=14,7)$. Na amostra predominou o sexo feminino, casado e analfabeto, $73,9 \%$ relataram baixa incerteza, $50,4 \%$ referiram qualidade de vida muito boa e $52,9 \%$ apresentaram valores de PA Sistólica $>140 \mathrm{mmHg}$. A qualidade de vida correlacionou-se significativamente com a pressão arterial. Conclusão: é necessário realizar a detecção de incertezas nos pacientes para direcionar intervenções individualizadas, a fim de gerar enfrentamento positivo, melhorar a qualidade de vida e melhorar o controle da pressão arterial nessa população.

Descritores: Incerteza; Qualidade de Vida; Hipertensão.

\section{INTRODUCCIÓN}

La hipertensión arterial (HA)

es una afección donde la presión en los vasos sanguíneos es alta, con una tensión arterial igual o superior $a$ 140/90 mm Hg${ }^{1}$. La HA es un factor de riesgo que provoca complicaciones 
Hernández-Nicio A, Chávez-Vásquez JC, Gutiérrez-Valverde JM, Pimentel-Jaimes JA, Telumbre-Terrero JY, Juárez-Medina LL, et al.

cardiovasculares y causa 9,4 millones de muertes a nivel mundial al año². La prevalencia mundial de $H A$ en adultos de 18 años o más fue de $32 \%{ }^{3}$. En México, la Encuesta Nacional de Salud y Nutrición de Medio Camino menciona que la prevalencia de la HA fue de $25,5 \%$, mayor en mujeres que en hombres $(26,1 \%$ vs $24,9 \%$, respectivamente). En Oaxaca, la prevalencia de HA fue del $11,7 \%{ }^{4}$.

La HA es una enfermedad que ocasiona alteraciones en la calidad de vida de aquellos que la sobrellevan gran parte de su vida, en donde se presentan sentimientos de miedo, temor, incertidumbre, impotencia y desesperanza $a^{5}$. La incertidumbre ha sido muy estudiada en personas con diagnóstico de cáncer, considerada como la incapacidad de para identificar los eventos relacionados a su enfermedad 6 .

La incertidumbre tiene un impacto negativo en la calidad de vida (CV) de las personas, debido a que la incertidumbre causa estrés, angustia y miedo ${ }^{7,8}$. La incertidumbre disminuye con el tiempo, pero incrementa con la aparición de síntomas que son impredecibles $e$ inconsistentes y la falta de información sobre su enfermedad para identificar los cambios a futuro ${ }^{8}$ y la baja escolaridad ${ }^{9,10}$. Si la incertidumbre permanece mucho tiempo provoca desorganización de ideas, inestabilidad emocional $y$ confusión $^{11}$. El apoyo social y la relación con el equipo de salud ayudan a mejorar la incertidumbre ante la enfermedad.

El impacto emocional que tienen la $\mathrm{HA}$ en los pacientes genera incertidumbre por el desconocimiento del proceso de enfermedad, el paciente presenta ideas de muerte, tristeza y por no saber las consecuencias a futuro ${ }^{12}$. Por lo tanto, la incertidumbre puede considerarse como un aspecto negativo en la $H A^{13}$, debido a que las personas con $H A$ presentan mayor nivel de ansiedad, preocupación y depresión en comparación con normotensos sin embargo, consideran que la HA no es tan peligrosa como la diabetes ${ }^{14,15}$.

En personas con $H A$, el cuidado no sólo debe enfocarse a retrasar la muerte, sino a mejorar la CV16. La CV es la satisfacción con las condiciones de la vida en el ámbito social, físico, mental y emocional, que puede ser valorada de manera subjetiva o por una tercera 
Hernández-Nicio A, Chávez-Vásquez JC, Gutiérrez-Valverde JM, Pimentel-Jaimes JA, Telumbre-Terrero JY, Juárez-Medina LL, et al.

persona ${ }^{17,18}$. No obstante, la Calidad de Vida Relacionada con la Salud (CVRS) se refiere a la satisfacción del individuo en cuanto a los aspectos físicos, sociales y psicológicos de su vida en la medida en que éstos se ven afectados por su salud ${ }^{19}$. En estudios con personas con HA la CV se ha reportado baja ${ }^{20}$. Sin embargo, las investigaciones sobre incertidumbre y calidad de vida están orientadas a diabetes, cáncer $e$ insuficiencia renal $^{21,22}$.

Evaluar la CVRS del paciente crónico es importante porque permite: conocer el impacto de la enfermedad; conocer mejor al enfermo, su evolución y su adaptación a la enfermedad. Para la profesión de enfermería el conocimiento acerca de la incertidumbre frente a la enfermedad y su asociación con la calidad de vida, permitiría profundizar en las necesidades de atención de la persona de cuidado, aportando información a la práctica clínica en el contexto de la HA y de los factores psicosociales que en ellas influyen ${ }^{22,23}$.

Además los profesionales de enfermería que tienen a su cargo pacientes con esta patología podrían incorporar estos aspectos psicosociales a la valoración y la intervención en enfermería en su plan de cuidados. Por lo tanto, el objetivo fue determinar la correlación entre el nivel de incertidumbre, la calidad de vida y la presión arterial en adultos con hipertensión de Santiago Pinotepa Nacional Oaxaca.

\section{MÉTODO}

El diseño del estudio fue transversal y correlacional ${ }^{24}$. La población estuvo constituida por pacientes hipertensos que acuden al centro de salud urbano de Santiago Pinotepa Nacional Oaxaca. El muestreo fue no aleatorio por conveniencia.

Los criterios de inclusión fueron pacientes con $H A$ con edad de 20 años y más, con diagnóstico mínimo de 6 meses y residentes de la ciudad de Pinotepa Nacional Oaxaca. Los criterios de exclusión fueron pacientes con alteraciones de las funciones cognitivas, que no hablen el idioma español, que no firmen el consentimiento informado, y que presenten otras enfermedades crónicas como cáncer $o$ diabetes mellitus. 
Hernández-Nicio A, Chávez-Vásquez JC, Gutiérrez-Valverde JM, Pimentel-Jaimes JA, Telumbre-Terrero JY, Juárez-Medina LL, et al.

En las mediciones realizadas, los datos sociodemográficos obtenidos fueron: Edad, sexo, estado civil y escolaridad. Los datos antropométricos obtenidos fueron el peso y la talla. La presión arterial se evaluó con un Baumanometro aerobico, con cinco minutos de espera y que el participante no haya tomado alguna bebida de cola, cafeína o haya fumado en los últimos 30 minutos.

La calidad de vida relacionada con la salud se midió con el cuestionario de la calidad de vida en hipertensión arterial $(\mathrm{CHAL})^{25}$, el instrumento está compuesto por 55 items agrupado en dos dimensiones: estado de ánimo (EA) con 36 ítems y manifestaciones somáticas (MS) con 19 ítems. El patrón de respuesta es de tipo Liker de 0 a 3. Las puntuaciones indican de 0-41 muy buena, 42-82 buena, 83-123 regular y 124- 165 mala. Un alfa de Cronbach de 0.89 y 0.96 respectivamente.

La variable incertidumbre se medió con la escala de incertidumbre frente a la enfermedad de Merle Mishel, recientemente traducida y validada al idioma español, es una escala compuesta por 23 ítems, agrupado en cuatro dimensiones: Tipología de los síntomas, factor coherencia de los hechos, capacidades cognitivas y autoridad con credibilidad $^{13}$. Las opciones de respuestas es en escala tipo Likert de 1 a 5 puntos. Nivel de incertidumbre bajo: menor de 59 puntos; nivel de incertidumbre regular: entre 59 a 87 puntos; nivel de incertidumbre alto: mayor de 87 puntos. Se obtuvo un alfa de Cronbach de 0.81.

Para la recolección de datos se obtuvo autorización y aprobación por parte del Centro de Salud para realizar esta investigación, se revisaron los expedientes clínicos y se obtuvieron la dirección del domicilio, posteriormente se realizó visita domiciliaria para invitarle a participar en el estudio, si aceptaba participar se hacía lectura del consentimiento informado y se le solicitaba firmara para participar en la investigación. Se iniciaba con el levantamiento de datos y al terminar, se le agradecía al paciente por su colaboración.

Los datos se capturaron y analizaron en el programa SPSS Statistics versión 22. Para el reporte de los datos, se utilizarán frecuencias y porcentajes para variables categóricas y medidas de tendencia central y dispersión para variables 
Hernández-Nicio A, Chávez-Vásquez JC, Gutiérrez-Valverde JM, Pimentel-Jaimes JA, Telumbre-Terrero JY, Juárez-Medina LL, et al.

continuas. El IMC se clasifico en $>18.5$ $<25$ peso normal, $\geq 25<30$ sobre peso $y \geq 30$ obesidad. Se utilizó la prueba de Anova de un factor para identificar diferencias entre más de dos grupos y se aplicó la prueba de correlación de Spearman.

\section{RESULTADOS}

La muestra estuvo integrada por 119 pacientes diagnosticado con $H A$, la edad promedio fue de 65.7 años $(D E=14.7)$. En la muestra predominó el sexo femenino, casado y respecto al ámbito educativo principalmente analfabeta (Tabla 1).

Tabla 1- Descripción de los participantes.

\begin{tabular}{lrr}
\hline $\begin{array}{l}\text { Datos } \\
\text { sociodemográficos }\end{array}$ & $f$ & $\%$ \\
Sexo & & \\
$\quad$ Mujer & 75.0 & 63.0 \\
$\quad$ Hombre & 44.0 & 37.0 \\
Estado Civil & & \\
$\quad$ Casado & 67.0 & 56.3 \\
$\quad$ Soltero & 17.0 & 14.3 \\
$\quad$ Unión Libre & 11.0 & 9.2 \\
$\quad$ Divorciado & 5.0 & 4.2 \\
$\quad$ Viudo & 19.0 & 16.0 \\
Escolaridad & & \\
$\quad$ Analfabeta & 52.0 & 43.7 \\
$\quad$ Primaria & 45.0 & 37.8 \\
$\quad$ Secundaria & 14.0 & 11.8 \\
$\quad$ Nivel Medio & 5.0 & 4.2 \\
$\quad$ Superior & & \\
$\quad$ Nivel Superior & 3.0 & 2.5 \\
\hline Nota: $\mathrm{n}=119$. & &
\end{tabular}

En relación a las variables de estudio el $67.2 \%$ de los participantes presentó sobrepeso/obesidad, con un promedio de IMC de $28.5(D E=6.8)$, el
$73.9 \%$ reportó una incertidumbre baja y $26.1 \%$ regular, el $50.4 \%$ señaló tener una muy buena calidad de vida y el $44.5 \%$ buena calidad de vida, el $52.9 \%$ presentó cifras de TA Sistólica $>140$ $\mathrm{mmHg}$ y $42.9 \%$ cifras de TA diastólica $>80 \mathrm{mmHg}$ (Tabla 2).

Tabla 2 - Descripción de las variables de estudios.

\begin{tabular}{lrrrr}
\hline Variable & \multicolumn{1}{c}{ M } & \multicolumn{1}{c}{ DE } & \multicolumn{1}{c}{ Min } & \multicolumn{1}{l}{ Max } \\
\hline $\begin{array}{l}\text { Calidad de } \\
\text { Vida }\end{array}$ & 41.4 & 21.3 & 3.0 & 100.0 \\
Incertidumbre & 51.4 & 11.1 & 32.0 & 83.0 \\
TA Sistólica & 136.2 & 12.6 & 110.0 & 170.0 \\
TA Diastólica & 81.0 & 11.6 & 60.0 & 110.0 \\
IMC & 28.5 & 6.8 & 16.6 & 55.3
\end{tabular}

Nota: $n=119$, Min = Mínimo; Max = Máximo; $D E=$ Desviación Estándar; $M=$ Media.

Para identificar las diferencias de calidad de vida e incertidumbre por las características sociodemográficas e IMC se utilizó t de student para dos grupos y Anova de un factor para más de dos grupos. No se encontraron diferencias significativas por sexo e IMC en calidad de vida e incertidumbre (datos no mostrados). Referente a la incertidumbre se encontró que las personas viudas presentan mayor incertidumbre, y en cuanto a la CVRS, de igual forma los viudos $y$ analfabetas presentan menor CVRS (Tabla 3). 
Hernández-Nicio A, Chávez-Vásquez JC, Gutiérrez-Valverde JM, Pimentel-Jaimes JA, Telumbre-Terrero JY, Juárez-Medina LL, et al.

Tabla 3 - Diferencia de calidad de vida incertidumbre por estado civil y escolaridad.

\begin{tabular}{|c|c|c|c|c|}
\hline Variable & M & $D E$ & $D_{e s t}$ & $p$ \\
\hline \multicolumn{5}{|l|}{ Calidad de Vida } \\
\hline \multicolumn{3}{|l|}{ Estado Civil } & 2.861 & .027 \\
\hline Casado & 38.6 & 17.7 & & \\
\hline Soltero & 36.9 & 26.3 & & \\
\hline Unión Libre & 45.0 & 18.2 & & \\
\hline Divorciado & 33.8 & 9.6 & & \\
\hline Viudo & 55.2 & 27.1 & & \\
\hline \multicolumn{3}{|l|}{ Escolaridad } & 3.721 & .007 \\
\hline Analfabeta & 49.5 & 21.5 & & \\
\hline Primaria & 34.6 & 19.7 & & \\
\hline Secundaria & 36.4 & 20.1 & & \\
\hline Nivel Medio & 38.6 & 13.2 & & \\
\hline \multicolumn{5}{|l|}{ Superior } \\
\hline Nivel Superior & 30.0 & 21.1 & & \\
\hline \multicolumn{3}{|l|}{ Estado Civil } & 3.416 & .011 \\
\hline Casado & 48.9 & 10.8 & & \\
\hline Soltero & 49.8 & 10.4 & & \\
\hline Unión Libre & 56.0 & 8.3 & & \\
\hline Divorciado & 55.6 & 8.8 & & \\
\hline Viudo & 57.8 & 11.7 & & \\
\hline \multicolumn{3}{|l|}{ Escolaridad } & 1.268 & .249 \\
\hline Analfabeta & 54.1 & 11.0 & & \\
\hline Primaria & 49.2 & 10.5 & & \\
\hline Secundaria & 49.7 & 11.8 & & \\
\hline Nivel Medio & 49.4 & 13.0 & & \\
\hline \multicolumn{5}{|l|}{ Superior } \\
\hline Nivel Superior & 49.3 & 12.8 & & \\
\hline
\end{tabular}

Nota: $\mathrm{n}=119, \quad M=$ Media, $\mathrm{DE}=$ Desviación Estándar, Prueba de Anova, $p=<.05$

Para cumplir con el objetivo general de determinar la relación entre el nivel de incertidumbre, la calidad de vida y la presión arterial en adultos con hipertensión arterial sistémica. Se utilizó la prueba de correlación lineal Sperman y los resultados muestra una correlación significativa y positiva entre la calidad de vida e incertidumbre, lo que indica que a mayor incertidumbre menor CVRS o viceversa, así como la presión arterial sistólica con la calidad de vida, señala que a mayor presión sistólica menor CVRS o viceversa (Tabla 4).

Tabla 4 - Correlación de las variables de estudio.

\begin{tabular}{lcccc}
\hline Variable & Sist & Diast & CVRS & Incert \\
Sist & - & & \\
Diast & $.557^{*}$ & - & & \\
CVRS & $.250^{*}$ & .003 & - \\
Incertidumbre & .123 & -.120 & $.483^{* *}$ & - \\
\hline Nota $n=119, C V=C a l i d a d$ de vida relacionada \\
a la salud, & Diast $=\quad$ diástolica, \\
Incert=incertidumbre, * $p<.05,{ }^{* *} p<.01$
\end{tabular}

\section{DISCUSIÓN}

La muestra predominio personas del sexo femenino, casados, analfabetas y con obesidad. La incertidumbre fue baja y la CVRS fue considerada muy buena, sin embargo en los valores de TA el mayor porcentaje presentaban cifras de hipertensión. A lo largo de la evolución de la hipertensión arterial las personas viven un proceso asintomático, las complicaciones y la preocupación por la enfermedad se presenta comorbilidades. Las personas no consideran a la HA como una enfermedad peligrosa, mientras que las personas no manifiesten síntomas asociados a la enfermedad o limitaciones para realizar sus actividades diarias consideraran que su CVRS es muy buena y no mostraran 
Hernández-Nicio A, Chávez-Vásquez JC, Gutiérrez-Valverde JM, Pimentel-Jaimes JA, Telumbre-Terrero JY, Juárez-Medina LL, et al. Incertidumbre y calidad...

preocupación por su cuidado o su salud $^{14,16}$.

En función a los hallazgos se encontró diferencias en la calidad de vida por estado civil y escolaridad. Las personas analfabetas presentan peor CVRS, esto se debe a que las personas no logren comprender la información que le proporcione por parte del personal de salud y no tenga los recursos económicos para cumplir con las dietas o medicamentos para mantener sus niveles de presión arterial y retrasen las comorbilidades. La baja CV se asocia con la dependencia del cuidado de algún familiar, por lo que las personas que han enviudado ya no cuentan con el cuidado de la pareja y presenten abandono ${ }^{26}$. Esta situación se presenta más en adultos mayores, quienes presentan mayores síntomas depresivos, abandono familiar y aislamiento social, aunado a las comorbilidades y complicaciones que se presentan al final de la vida.

También se encontraron diferencias de incertidumbre por estado civil. La teoría de incertidumbre señala que las personas casadas presentan menor incertidumbre debido al apoyo de pareja que presenta este grupo de personas, lo que ayudaría buscar información sobre su enfermedad y a no alarmarse de las complicaciones a futuro $^{7,8}$. A pesar de que no se presentaron diferencias significativas por escolaridad, las personas con mayor preparación educativa cuenta con mayores recursos cognitivos e intelectuales para afrontar las situaciones de la vida o la enfermedad, buscar información para conocer los síntomas de peligro o la integración a grupos de apoyo, por lo que las personas analfabetas presentan mayores niveles de incertidumbre.

Otro resultado importante fue que la incertidumbre se correlacionó con CVRS. La presencia y prolongación de manifestaciones somáticas y decaimiento del funcionamiento fisiológico y del estado de ánimo en la edad adulta son factores que se hacen presentes en la hipertensión arterial, lo que provoca que los pacientes manifiesten preocupación, estrés, ansiedad e incertidumbre ante su enfermedad y su vida. Esta situación se presenta a medida que la enfermedad evoluciona $y$ no se controla, debido a que los síntomas pueden agravarse, inconsistentes e 
Hernández-Nicio A, Chávez-Vásquez JC, Gutiérrez-Valverde JM, Pimentel-Jaimes JA, Telumbre-Terrero JY, Juárez-Medina LL, et al.

impredecibles, con mayor o menor magnitud, principalmente si se relacionan con comorbilidades ${ }^{7,8,11}$. Educar a los pacientes a reconocer los síntomas de alarma sobre su enfermedad, podrá ayudar a disminuir sus niveles de incertidumbre.

Por último, se correlacionó la CVRS con la presión sistólica, lo cual ha sido reportado en otros estudios ${ }^{27}$, las personas que tienen inadecuado control muestran cuatro veces mayor probabilidad de tener menor CV. De la misma manera, las personas que ingresan a urgencias con diagnóstico previo de HA presentan menor CV relacionado con comorbilidades cardiovasculares $^{20}$. Cuando se cuenta con antecedentes como infarto al miocardio 0 accidentes cerebrovasculares, situaciones que manifiestan de manera repentina, puede generar limitaciones físicas que afecten de manera negativa su función social, rol físico, emocional y su vitalidad.

\section{CONCLUSIÓN}

A partir de los resultados del presente estudio el profesional de enfermería puede plantear intervenciones respecto a la educación de las personas que viven con el diagnostico de HA con la finalidad de potencializar la concientización sobre dicha enfermedad. También se considera necesario realizar detección de la incertidumbre en este tipo de población para dirigir intervenciones individualizadas y así generar un afrontamiento positivo que impacte en la adaptación del paciente ante su enfermedad.

Lo anterior puede contribuir a que se logre un mejor control de las cifras tensionales de la presión arterial que repercuten directamente en la calidad de vida. Asimismo, puede resultar benéfico integrar grupos de ayuda mutua en el primer nivel de atención a la salud, donde los pacientes puedan interactuar con otras personas con el mismo padecimiento y poder compartir sus experiencias, ya que, se observó que el estado civil influye en la calidad de vida, lo cual podría explicar que si la persona se siente apoyada, mejor será su calidad de vida.

Las limitaciones del estudio fue el tamaño de muestra, lo que impide hacer generalizaciones significativas a partir de los datos y así poder extrapolar los resultados a 
Hernández-Nicio A, Chávez-Vásquez JC, Gutiérrez-Valverde JM, Pimentel-Jaimes JA, Telumbre-Terrero JY, Juárez-Medina LL, et al. Incertidumbre y calidad...

poblaciones más grandes, también, debido al diseño del estudio, no se puede establecer criterios de causaefecto. Las futuras investigaciones, además de incrementar el tamaño de muestra; deberán contemplar la inclusión de variables relacionadas con el tratamiento farmacológico y estudios con abordaje cualitativo para mayor comprensión del fenómeno de la incertidumbre en los pacientes con hipertensión arterial.

\section{REFERENCIAS}

1. Secretaria de Salud. Norma Oficial Mexicana NOM-043-SSA2-2012. Servicios básicos de salud. Promoción y educación para la salud en materia alimentaria. Criterios para brindar orientación. Diario Oficial de la Federación. 2013. Disponible en: http: / / www.cndh.org.mx/DocTR/2 016/JUR/A70/01/JUR-20170331NOR37.pdf

2. Organización Mundial de la Salud. Información general sobre hipertensión en el mundo. 2013. Disponible en: http:/ / apps.who.int/iris/bitstream /10665/87679/1/WHO_DCO_WHD_2 013.2_spa.pdf
3. Reboussin DM, Allen NB, Griswold ME, Guallar E, Hong Y, Lackland DT, et al. Systematic review for the 2017

ACC/AHA/AAPA/ABC/ACPM/AGS/AP hA/ASH/ASPC/NMA/PCNA guideline for the prevention, detection, evaluation, and management of high blood pressure in adults: a report of the American College of Cardiology/American Heart Association Task Force on Clinical Practice Guidelines. J Am Coll Cardiol. 2018; 71(19): 2176-2198.

4. Instituto Nacional de Salud Pública. Encuesta nacional de salud y nutrición: Resultados por entidad federativa. 2012. Disponible en: https://www.insp.mx/images/stori es/ENSANUT/sureste/OaxacaOCT.pdf

5. Vargas-Escobar LM. Marco para el cuidado de la salud en situaciones de enfermedad crónica. Investig Enferm Imagen Desarr. 2010; 12(1):74-94.

6. Zhang $Y$. Uncertainty in Illness: Theory Review, Application, and Extension. Oncol Nurs Forum. 2017; 44(6):645-649.

7. Mishel MH. Uncertainty in Illness. J Nurs Scholarsh. 1988; 20(4):22532. 
Hernández-Nicio A, Chávez-Vásquez JC, Gutiérrez-Valverde JM, Pimentel-Jaimes JA, Telumbre-Terrero JY,

8. Mishel MH. What do We Know about Uncertainty in Illness?. J Jpn Soc Nurs Res. 2006; 29(1):19-26.

9. Johnson Castro MI, Hurtado Arenas P. La incertidumbre frente a la enfermedad en adultos mayores con diagnóstico de cáncer. Cienc Enferm. 2017; 23(1):57-65.

10. Montalvo Prieto AA, Fajardo Rocha $H$, Angulo Yepes TB, Flórez Navas DE, Caffroni Monterroza RA, Fajardo Torres YM. Condiciones sociodemográficas y nivel de incertidumbre en mujeres ante el diagnóstico de cáncer de mama. Hacia promoc. Salud. 2016; 21(2):114-126.

11. Mishel MH. Reconceptualization of the uncertainty in illness theory. $J$ Nurs Scholarsh.1990; 22(4): 256262.

12. Gonzáles Gonzáles N, Tinoco García AM, Benhumea Gonzáles LE. Salud mental y emociones en pacientes con enfermedades crónico-degenerativas. Un acercamiento a la diabetes mellitus tipo 2. Espacios Públicos. 2011; 14(32):258-279.

13. Gómez-Palencia IP, Castillo-Ávila IY, Alvis-Estrada LR. Incertidumbre en adultos diabéticos tipo 2 a partir de la teoría de Merle Mishel. Aquichan. 2015; 15(2):210-218.

14. Segura Barrios IM, Barrera Ortiz L. Un llamado a Enfermería para responder al cuidado de la salud de las personas en situación de enfermedad crónica por su impacto en su calidad de vida. Salud Uninorte. 2016; 32(2): 228243.

15. Velázquez Díaz $M$, Espinoza Gonzáles F, Martínez Medina MP, Padrós Blázquez F. Preocupación patológica en hipertensos en comparación con normotensos en población michoacana (México). Acta Univ. 2016; 26(2):70-76.

16. Gómez Gómez MR, García Reza C, Gómez Martínez V, Mondragón Sánchez PB. Calidad de vida en pacientes que viven con hipertensión arterial sistémica. Rev Mex Enferm Cardiol. 2011; 19(1):7-12.

17. Schwartzmann L. Calidad de Vida relacionada con la salud: aspectos conceptuales. Cienc Enferm. 2003; 9(2): 9-21.

18. Urzúa MA, Caqueo-Urízar A. Calidad de vida: una revisión teórica del concepto. Ter Psicol. 2012; 30(1):61-71. 
Hernández-Nicio A, Chávez-Vásquez JC, Gutiérrez-Valverde JM, Pimentel-Jaimes JA, Telumbre-Terrero JY, Juárez-Medina LL, et al. Incertidumbre y calidad...

19. Urzúa MA. Calidad de Vida relacionada con la Salud: elementos conceptuales. Rev Med Chile. 2010; 138(3): 358-365.

20. Ortunio M, Guevara H, Cardozo R. Calidad de vida en pacientes con hipertensión. Rev Informe Med. 2013; 15(2):45-50.

21. Arreguin Sánchez L, Morales Rodríguez MC, Bonilla Luis ML, Soriano y Sotomayor MM, Tlalpan Hernández RMT, Pérez Badillo V. Incertidumbre y calidad de vida en mujeres y hombres afectados por cáncer. Enfermería (Montev). 2012; 1(2):77-83.

22. Torres AA, Sanhueza OA. Modelo estructural de enfermería de calidad de vida e incertidumbre frente a la enfermedad. Cienc Enferm. 2006; 12(1): 9-17.

23. Vinaccia S, Orozco LM. Aspectos psicosociales asociados con la calidad de vida de personas con enfermedades crónicas. Divers.:
Perspect. Psicol. 2005; 1(2): 125137.

24. Hernández R, Fernández C, Baptista MDP. Metodología de la investigación. México: McGrawHill; 2014.

25. Dalfó i Baqué A, Badia i Llach X, Roca-Cusachs A. Cuestionario de calidad de vida en hipertensión arterial (CHAL). Aten Primaria. 2002; 29(2):114-121.

26. Nolasco Monterroso C, Navas Santos L, Carmona Moriel CE, López Zamorano MD, Santamaria Olmo R, Crespo Montero R. Análisis de los factores asociados a la calidad de vida del paciente hipertenso. Enferm Nefrol. 2015; 18(4):282-289.

27. Serrano Garzón E, Michel Olague $M B$, Frías Mora R, Álvarez Gutiérrez OA, Frías Serrano RG. Calidad de vida en pacientes con hipertensión arterial. Rev Med. 2018; 9(2):146-151.

Conflito de interesses: Os autores declaram não haver conflito de interesses.

Participação dos autores:

- Concepção: Hernández-Nicio A, Chávez-Vásquez JC, GutiérrezValverde JM, Pimentel-Jaimes JA, Telumbre-Terrero JY, JuárezMedina, Mendoza-Catalán G.

- Desenvolvimento: Hernández-Nicio A, Chávez-Vásquez JC, Gutiérrez-Valverde JM, Pimentel-Jaimes JA, Telumbre-Terrero JY, Juárez-Medina, Mendoza-Catalán G.

- Redação e revisão: Hernández-Nicio A, Chávez-Vásquez JC, Gutiérrez-Valverde JM, Pimentel-Jaimes JA, Telumbre-Terrero JY, Juárez-Medina, Mendoza-Catalán G. 
Hernández-Nicio A, Chávez-Vásquez JC, Gutiérrez-Valverde JM, Pimentel-Jaimes JA, Telumbre-Terrero JY, Juárez-Medina LL, et al. Incertidumbre y calidad...

Como citar este artigo: Hernández-Nicio A, Chávez-Vásquez JC, Gutiérrez-Valverde JM, Pimentel-Jaimes JA, Telumbre-Terrero JY, Juárez-Medina LL, et al. Incertidumbre y calidad de vida en pacientes con hipertensión. J Health NPEPS. 2019; 4(2):240-252.

Submissão: 04/06/2019

Aceito: $20 / 11 / 2019$

Publicado: 01/12/2019 